\title{
Dilemas en el quehacer de las bibliotecas universitarias argentinas en torno al acceso y difusión de la literatura científica
}

\section{Marcela Fushimi}

Universidad Nacional de La Plata. Facultad de Humanidades y Ciencias de la Educación. Biblioteca Profesor Guillermo Obiols (BIBHUMA) y Departamento de Bibliotecología. Universidad Nacional de La Plata. Instituto de Investigaciones en Humanidades y Ciencias Sociales (IdIHCS. FaHCE. UNLP-CONICET). Argentina I mfushimi@fahce.unlp.edu.ar / https://orcid.org/oooo-0oo2-7266-8216

\author{
Mónica G. Pené \\ Universidad Nacional de La Plata. Facultad de Humanidades y Ciencias de la Educación. Biblioteca Profesor Guillermo Obiols \\ (BIBHUMA) y Departamento de Bibliotecología. Universidad Nacional de La Plata. Instituto de Investigaciones en Humanidades y \\ Ciencias Sociales (IdIHCS. FaHCE. UNLP-CONICET). Argentina I mpene@fahce.unlp.edu.ar / https://orcid.org/oooo-0002-0257-7634
}

\section{Carolina Unzurrunzaga}

Universidad Nacional de La Plata. Facultad de Humanidades y Ciencias de la Educación. Departamento de Bibliotecología. Universidad Nacional de La Plata. Instituto de Investigaciones en Humanidades y Ciencias Sociales (IdIHCS. FaHCE. UNLP-CONICET). Argentina | cunzu@fahce.unlp.edu.ar / https://orcid.org/oooo-0002-4383-0085

\author{
Ana María Sanllorenti \\ Universidad de Buenos Aires. Facultad de Ciencias Exactas y Naturales. Biblioteca Central Dr. Federico Leloir Universidad \\ Nacional de Mar del Plata. Facultad de Humanidades. Departamento de Ciencia de la InformaciónUniversidad Nacional de La \\ Plata. Instituto de Investigaciones en Humanidades y Ciencias Sociales (IdIHCS. FaHCE. UNLP-CONICET). Argentina I \\ asanllorenti@bl.fcen.uba.ar / https://orcid.org/oooo-0002-9639-4504
}

\begin{abstract}
Resumen
En este ensayo nos proponemos reflexionar acerca del rol del bibliotecario universitario como agente difusor y proveedor de acceso a la información científica en Argentina. Para ello, exponemos una serie de problemáticas que se plantean en la práctica profesional y que nos generan dilemas: la tensión entre el derecho de acceso a la información y la propiedad intelectual; nuestro imaginario sobre el acceso abierto y la forma en que lo difundimos ante la comunidad académica, frente a las reglas del campo científico que deben seguir los investigadores para progresar en sus carreras; y la vinculación con los proveedores comerciales frente a nuestras convicciones acerca del camino a seguir para alcanzar la democratización del acceso al conocimiento científico. Luego de los planteos y discusiones sobre estos dilemas, trayendo al diálogo experiencias propias y de colegas, así como halladas en la literatura consultada, finalizamos recapitulando algunos aspectos para repensar nuestro rol en la academia, poniendo el foco de atención en nuestra función social.
\end{abstract}

Palabras clave

Acceso a la información científica Biblioteca universitaria Acceso abierto Comunicación científica Rol bibliotecario 


\section{Keywords}

Access to scientific information University Library

Open access

Scientific communication

Librarian role

\section{Dilemmas of Argentine university libraries regarding the access and dissemination of scientific literature}

\section{Abstract}

In this essay we reflect on the role of the university librarian as a disseminating agent and provider of access to scientific information in Argentina. First, we present problems that arise in professional practice and generate dilemmas: the tension between the right of access to information and intellectual property; our imaginary on open access and the way in which we disseminate it into the academic community, facing the rules of the scientific field that researchers must follow to progress in their careers; and the relationship with commercial providers in front of our convictions about the way forward to achieve the democratization of access to scientific knowledge. After the debate on these dilemmas, where we brought to the dialogue our own experiences and those of colleagues, as well as those found in the consulted literature, we discuss some aspects to rethink our role in the academy, putting the focus of attention on our social function.

Artículo recibido: 25-05-2020 Aceptado: 23-10-2020

"No hay justicia alguna en obedecer leyes injustas.

Es tiempo de salir a la luz y en la gran tradición de la desobediencia civil, declarar nuestra oposición a este robo privado de la cultura pública"

(Swartz, 2008)

\section{Introducción ${ }^{1}$}

En este ensayo reflexionamos acerca de la misión de las bibliotecas universitarias y especializadas en dos de sus funciones tradicionales. La primera se refiere a la provisión de la bibliografía necesaria para el desarrollo de las actividades de enseñanza e investigación, normalmente, a través de la formación y organización de un fondo documental acorde con las necesidades de la comunidad a la que ofrece servicios. Esta función está atravesando un cambio radical debido al tránsito del mundo impreso al digital: pasamos de la adquisición y posesión del material al pago de suscripciones para ofrecer acceso a recursos que la Biblioteca ya no posee ni organiza, ni tampoco selecciona como antes. La segunda se vincula con las tareas de reunión, registro, preservación y difusión de las producciones generadas por la comunidad de pertenencia, que en el nuevo milenio se manifiestan mediante la construcción de bibliotecas y repositorios digitales que albergan y difunden la memoria institucional. Estas dos funciones -acceso y difusión- han sido pilares de la tarea bibliotecaria desde siempre y continúan hoy día en el contexto digital. Sin embargo, diversos factores las afectan, algunos de larga data y otros de más reciente aparición: la preponderancia de la cuestión patrimonial en materia del derecho de autor ante la ausencia de excepciones para las instituciones culturales, la aparición de nuevos actores que proveen acceso gratuito (aunque no abierto) a la literatura requerida, la escasa inserción en la comunidad de los repositorios digitales gestionados por bibliotecas, la omnipresencia de los proveedores comerciales de contenidos digitales autoproclamados "de calidad", las reglas del campo científico y las necesidades de los investigadores, entre otras, que cuestionan nuestro papel en este ecosistema y nos obligan a repensar prácticas y servicios de información. 
Durante nuestro trabajo en bibliotecas de universidades argentinas de gestión pública, así como en intercambios con distintos colegas, observamos, en diferente grado y manifestación, una serie de problemáticas que frecuentemente se nos plantean como dilemas en el quehacer bibliotecario. La primera es la histórica y tensa situación entre el derecho de acceso a la información y la propiedad intelectual. La segunda, nuestro imaginario sobre el acceso abierto, que se entremezcla con las utopías de antaño sobre el acceso a la bibliografía universal, y la forma en que difundimos esta situación dentro de la comunidad académica, frente a las exigencias que las y los investigadores deben acatar para sobrevivir y obtener reconocimiento en sus carreras. Y la tercera, que nos tensiona ideológicamente, se relaciona con la vinculación que debemos mantener con proveedores comerciales para satisfacer las necesidades informativas de nuestra comunidad usuaria, que si bien de hecho parece ser una convivencia necesaria, se contrapone a nuestras convicciones acerca del camino a seguir para alcanzar la democratización del acceso al conocimiento científico y a su concepción como bien común.

En el presente trabajo reflexionamos acerca del rol del bibliotecario universitario como agente difusor y proveedor de acceso a la información científica. Para ello, discutimos en torno a los tres dilemas enunciados anteriormente y traemos al diálogo experiencias propias y ajenas encontradas en la literatura especializada acerca de distintas cuestiones que condicionan el acceso a la información y nos interpelan como profesionales.

\section{El derecho de acceso a la información y la propiedad intelectual: tensiones y dificultades}

Entre los derechos de acceso a la información, al conocimiento y al goce de los beneficios de la ciencia y la cultura, reconocidos en declaraciones y pactos internacionales suscriptos por Argentina y asentados en la Constitución nacional ${ }^{2}$, y la propiedad intelectual que incluye al derecho de autor y que se expresa en varios corpus normativos a nivel nacional e internacional -también reconocidos en la Constitución-, se despliegan múltiples tensiones que, lejos de resolverse, en las últimas décadas han crecido en intensidad en ámbitos diferentes.

En los últimos 40 años, tal como señala Correa (2009: 130), se ha producido una expansión del proteccionismo en todos los ámbitos relacionados con el conocimiento y la cultura que no conoce antecedentes, y que "ha alterado dramáticamente la relación entre los intereses del público y los de quienes detentan derechos de propiedad intelectual", lo que se observa en la extensión de los plazos de protección, el patentamiento de seres vivos y las medidas tecnológicas de protección, entre otros hechos. El área de la comunicación de la ciencia presenta un particular escenario para el desarrollo de estas tensiones. A la apropiación sin pago de los resultados de la investigación científica que sostienen las instituciones públicas y privadas por parte de las editoriales comerciales en el proceso de publicación de artículos, se suma el desconocimiento que los investigadores tienen de sus propios derechos de autor. En el afán por publicar, firman sin más la cesión exclusiva de sus derechos patrimoniales, lo que les impide reutilizar legalmente los artículos en actividades de enseñanza o investigación. A lo sumo pueden hacerlo luego de prolongados plazos de embargo impuestos por las editoriales. En el caso de Argentina, la Ley nacional 26.899 de Creación de Repositorios Digitales Institucionales de Acceso Abierto ${ }^{3}$, sancionada por el Senado de la Nación en diciembre de 2013 y reglamentada a fines de 2016, establece que los investigadores deben depositar sus trabajos en repositorios institucionales a los 6 meses de editados o aprobados, lo que suele entrar en contradicción
2. Nos referimos a la Declaración Universal de los Derechos Humanos (1948) y al Pacto Internacional de Derechos Económicos, Sociales y Culturales (1966).
3. Esta legislación determina que la literatura científica y los datos primarios de las investigaciones que son financiadas con fondos públicos deben estar en acceso abierto a través de repositorios digitales que las instituciones del sistema científico y tecnológico nacional estarán obligadas a crear y gestionar. Asimismo, exige a los investigadores el depósito de sus producciones y de los datos primarios que generan en estos repositorios. 
4. ADPIC/ TRIPS establece en el Artículo 8 "Principios" inciso 1: "Los Miembros, al formular o modificar sus leyes y reglamentos, podrán adoptar las medidas necesarias para proteger la salud pública y la nutrición de la población, o para promover el interés público en sectores de importancia vital para su desarrollo socioeconómico y tecnológico, siempre que esas medidas sean compatibles con lo dispuesto en el presente Acuerdo." con los plazos de embargo de las editoriales o con las prohibiciones completas de algunas de ellas (Ley 26.899, 2013).

En este sentido, cabe preguntarnos cuál es la postura que debemos adoptar como profesionales de la información que, en buena medida, hemos estado a la cabeza de la construcción de repositorios y del acceso abierto en el país: ¿respetar en forma estricta los acuerdos de cesión exclusiva de derechos que firman los investigadores con las editoriales y que se encuentran protegidos por la legislación internacional de propiedad intelectual y los plazos de embargo establecidos por los editores, o poner a disposición de la sociedad el conocimiento que se ha producido y sostenido con financiamiento público, tal como lo indica la Ley 26.899? El grupo de investigadoras que ha preparado este trabajo no tiene dudas de que debemos estar del lado del acceso abierto al conocimiento científico y priorizar el cumplimiento de la ley argentina, realizando todos los intentos, capacitaciones, sensibilizaciones en la comunidad académica para contribuir a que la mayor parte de la producción científica de nuestras instituciones se difunda lo más ampliamente posible. Esta posición se ve respaldada en varios párrafos del Código de Ética de IFLA para bibliotecarios y trabajadores de la información (IFLA, 2012):

La misión principal de los bibliotecarios y otros trabajadores de la información es garantizar el acceso a la información para todos, para el desarrollo personal, educación, enriquecimiento cultural, ocio, actividad económica y participación informada para la mejora de la democracia (...) El interés de los bibliotecarios y otros trabajadores de la información es proporcionar a los usuarios el mejor acceso posible a la información e ideas en cualquier medio o formato. Esto incluye el apoyo a los principios del acceso abierto, código abierto, y licencias abiertas.

Las tensiones entre la propiedad intelectual y el derecho de la sociedad a recibir los beneficios de los resultados de la investigación científica, son de una relevancia tal que se han visto reflejadas en los textos de algunos tratados internacionales que habilitan excepciones y limitaciones a la propiedad intelectual. En tiempos de la pandemia del coronavirus COVID-19 cabe recordar que el Acuerdo sobre los aspectos de los derechos de propiedad intelectual relacionados con el comercio (ADPIC/ TRIPS) al que Argentina ha adherido, habilita a los gobiernos de los países miembros de la Organización Mundial del Comercio a que adopten medidas para proteger la salud pública. Eso les permite hacer uso de las flexibilidades previstas a ese efecto, como por ejemplo, fabricar genéricos sin considerar las patentes que controlan los medicamentos, mediando algunos procedimientos requeridos ${ }^{4}$.

En la misma dirección, una coalición de más de 160 instituciones que representan a educadores, bibliotecas, archivos, museos, académicos de la propiedad intelectual y defensores del acceso a medicamentos de 199 países, dirigió una nota en abril de 2020 al Dr. Francis Gurry, Director General de la Organización Mundial de la Propiedad Intelectual (OMPI), que circuló y cosechó numerosas adhesiones a nivel internacional. En esta carta se solicitó a la OMPI que ante la pandemia: 1) impulse a los Estados Miembros a utilizar las flexibilidades que permiten el uso de trabajos protegidos para la educación en línea, la investigación y los usos experimentales, y para intereses públicos vitales como el acceso a la salud y la cultura; 2) reclame a los propietarios de los derechos el retiro de las restricciones de licencias que inhiben la educación remota, la investigación (incluyendo textos y minería de datos y proyectos de ingeniería artificial) y el acceso a la cultura en forma transfronteriza; 3 ) promueva varias medidas para eliminar barreras a la fabricación global, distribución y venta de productos potencialmente efectivos para detectar, prevenir y tratar el COVID-19, y 4) respalde los derechos de los países para promulgar y utilizar las excepciones al secreto comercial y otros derechos de propiedad intelectual a fin de facilitar un mayor acceso 
a la información de fabricación, y a todos los recursos que promuevan la disposición universal y equitativa de los medicamentos y tecnologías médicas relacionadas con la enfermedad (Open Letter on Intellectual Property and COVID-19 to the Director General of WIPO, 3 abril de 2020).

En los ejemplos anteriores mencionamos algunas de las flexibilidades existentes en la legislación internacional. Lamentablemente, en nuestro país la Ley 11.723 -vigente con modificaciones desde 1933- no incluye excepciones a favor de las bibliotecas, archivos o museos, ni tampoco en beneficio de la investigación y la educación. Como bibliotecarios debemos respetar el derecho de los creadores y de las obras protegidas por el derecho de propiedad intelectual pero, al mismo tiempo, bregar por la inclusión de excepciones ${ }^{5}$ que nos permitan cumplir a cabalidad nuestra misión de dar acceso al conocimiento y preservarlo, lo que también se encuentra formulado en varios párrafos del Código de Ética de IFLA. Un hecho a destacar es la iniciativa del abogado Alejandro Butler, respaldada por el Grupo Bibliotecario sobre Acceso a la Información y Propiedad Intelectual (G-BAIPI), quien en abril de 2020 presentó ante la Presidencia de la Nación una petición a fin de que se libere el derecho de acceso a obras protegidas por derecho de autor mediante la autorización a bibliotecas, archivos y museos, instituciones, centros y establecimientos científicos, culturales, artísticos y de enseñanza del país, a los efectos de suministrar el material que los usuarios requieran durante el tiempo que dure la emergencia sanitaria por la pandemia. ${ }^{6}$

Asimismo, nuestro aporte como colectivo profesional debe proyectarse en la elaboración de políticas institucionales de acceso abierto y de instrumentos para la gestión del derecho de autor en los repositorios, tales como licencias y addenda a los acuerdos de edición, cuya aplicación posibilita un trato equitativo entre investigadores e instituciones responsables, en los que ambas partes asumen deberes y derechos. Estos instrumentos institucionales incluyen el tratamiento de la propiedad intelectual evitando las cesiones exclusivas de derechos por parte de los autores y habilitando a las instituciones a que puedan registrar y ofrecer libremente lo que se produce en su marco (Sanllorenti, Pelaya y Williman, 2012). Al respecto, el Código de Ética de IFLA sostiene:

Los bibliotecarios y otros trabajadores de la información negocian las condiciones más favorables para el acceso a las obras en representación de sus usuarios y buscan garantizar que el acceso no se vea innecesariamente impedido $\mathrm{u}$ obstaculizado por la forma de administración de las leyes de propiedad intelectual y que las licencias no invaliden o anulen las excepciones para las bibliotecas incluidas en la legislación nacional. (IFLA, 2012)

Es permanente en el quehacer de las bibliotecas universitarias -y de todas las bibliotecas- la aparición de situaciones en las que se manifiestan los conflictos entre el derecho de la sociedad de acceder a la información, el conocimiento y la cultura por un lado, y la propiedad intelectual por otro. Las autoras de este artículo consideramos que, en tales disputas, las bibliotecas y los bibliotecarios deben asentar su prioridad en la defensa de los derechos de acceso, al tiempo de asumir la custodia del derecho moral de los autores a ser reconocidos y citados como tales.

\section{El discurso bibliotecario del acceso abierto y las prácticas científicas}

Cuando se sentaron las bases del movimiento de acceso abierto con las conocidas declaraciones $\mathrm{BBB}^{7}$, los bibliotecarios, como actores implicados en el proceso de comunicación científica, nos involucramos en el desarrollo de la denominada vía
5. En tres oportunidades, entre 2010 y 2015, la Subcomisión de Propiedad Intelectual, Acceso a la Información y Libertad de Expresión de la Asociación de Bibliotecarios Graduados de la República Argentina (ABGRA), presentó proyectos de ley para incluir esas excepciones en la legislación. Todos perdieron estado parlamentario al no lograr ser tratados en el Congreso. El último proyecto puede consultarse en <https:// hcdn.gob.ar/proyectos/proyecto. jsp? $\exp =5792-D-2015>$. El actual Grupo Bibliotecario sobre Acceso a la Información y la Propiedad Intelectual (G-BAIPI) continúa trabajando con los mismos objetivos.

6. Butler, A. (2020) Petición al Poder Ejecutivo Nacional. Propuesta de ampliación del derecho de acceso a las obras protegidas por derecho de autor para la educación, la investigación y el esparcimiento a distancia. <https://drive. google.com/file/d/1MrFfKCO1Sg YOfwMxkE8ML1NOsKwPg2GX/ view? $\mathrm{usp}=$ drivesdk $>$ La propuesta fue presentada al Poder Ejecutivo por cuanto en abril de 2020 -fecha de su ingreso- no se encontraba aún en funcionamiento el Congreso de la Nación Argentina.
7. Nos referimos a la Iniciativa de Budapest de Acceso Abierto (BOA 2002), la Declaración de Bethesda sobre la publicación de acceso abierto (2003) y la Declaración de Berlín sobre el Acceso Abierto al conocimiento en Humanidades y Ciencias Sociales (2003). En América Latina contamos con la Declaración de Salvador sobre Acceso Abierto: la perspectiva del mundo en desarrollo (2005). 
8. Article Processing Charges o costo de procesamiento de artículos, es una tasa que cobran las editoriales a los autores para publicar su artículo en acceso abierto, en una revista que puede ser abierta o cerrada. verde -el autoarchivo- construyendo repositorios digitales en nuestras instituciones. Nos apropiamos rápidamente del discurso del acceso abierto y nos transformamos en agentes activos del cambio propuesto (Fushimi, 2018b). La filosofía intrínseca del movimiento coincidía con los objetivos tradicionales de la profesión bibliotecaria y ampliaba las posibilidades para cumplir nuestro rol social, ubicando a la profesión en un espacio más visible, y hasta rememorando la utopía del control bibliográfico universal enunciada por Otlet y La Fontaine a finales del siglo XIX. Además de la construcción y mantenimiento de repositorios, sumamos un rol activo y entusiasta a favor del acceso abierto mediante actividades de promoción, talleres, cursos para fomentar el autoarchivo y el apoyo de marcos normativos que lo promueven, como lo es, en el caso de Argentina, la ley 26.899.

Sin embargo, la realidad de los investigadores está ceñida por los intereses y las pujas del campo académico para obtener promociones y financiamiento, por lo que están inmersos en una cultura evaluativa que utiliza como parámetro principal la cantidad y calidad de las publicaciones que realizan. En esta se le ha otorgado a las revistas un rol protagónico, así como también a los sistemas de indexación para legitimación de los resultados. Docentes, investigadores y becarios, que son parte de nuestras comunidades académicas, se ven demandados ante la exigencia de publicar los resultados de las investigaciones con formato de artículo en revistas científicas "prestigiosas" y rehenes de un sistema que trata al conocimiento como mercancía. En los últimos años, con el avance de las políticas de acceso abierto en los diferentes países, hemos visto como las editoriales que ejercen un oligopolio en el sector (Larivière, Haustein y Mongeon, 2015) adecuaron sus prácticas y modelos de negocios para sostener su lugar de poder en el proceso de la comunicación científica. El caso paradigmático fue el surgimiento de las llamadas revistas híbridas u open choice, que no son otra cosa que revistas tradicionales cerradas en las cuales el autor puede liberar su artículo pagando una tasa de publicación, conocida por su sigla en inglés como $\mathrm{APC}^{8}$. En tanto, las restricciones editoriales para permitir el autoarchivo de artículos en repositorios institucionales fueron creciendo y aumentando en complejidad, lo que generó confusión en los autores acerca de las versiones permitidas, los plazos de embargo, los usos admitidos, y también, temores acerca de infringir compromisos previamente asumidos.

Asimismo, nos encontramos con que las formas en las que buscan y obtienen la información que necesitan para su trabajo los aleja cada vez más de los artefactos bibliotecarios tradicionales. La aparición de otras infraestructuras de información como las redes sociales académicas (ResearchGate y Academia) y los sitios de descarga gratuita (Sci-Hub y LibGen) que presentan mejores funcionalidades, diseños intuitivos y más atractivos, responden mejor a sus necesidades. Trabajos recientes dan cuenta del uso creciente de estos servicios por parte de la comunidad universitaria para acceder a la literatura científica (Bohannon, 2016; Machin-Mastromatteo, Uribe-Tirado y Romero-Ortiz, 2016), y en el caso de las redes sociales también como canal para difundir, compartir e intercambiar la producción propia con colegas de la misma disciplina (Laakso y Lindman, 2016; Borrego, 2017; Unzurrunzaga, 2017 y Miguel, González y Ortiz-Jaureguizar, 2018).

Ante esta situación nos encontramos realizando actividades de difusión que suelen tener escasa llegada a sus destinatarios, lo que nos hace pensar si usamos los canales adecuados para llegar hasta ellos, si estamos utilizando las frases e ideas correctas, si no habrá cierta desconexión entre la información que se pretende difundir y la realidad de las personas involucradas, transmitiendo un mensaje ingenuo, incompatible con las prácticas de los interesados. Este problema, que va de la mano de una falta de interés por el mensaje que transmitimos, se traduce en un bajo porcentaje de autoarchivo de trabajos en los repositorios. Si bien los investigadores reconocen la 
importancia del acceso abierto para la democratización del conocimiento científico $\mathrm{y}$, en muchos casos, son conscientes del rol que juegan las grandes editoriales en la apropiación gratuita del conocimiento producido en las instituciones científicas y en el establecimiento de estándares internacionales de prestigio académico, se observa una actitud de aceptación del statu quo, seguramente reforzada por la necesidad de publicar para poder continuar con sus carreras. En resumen, realizamos la difusión de nuestros repositorios en formas desituadas de su contexto, con mensajes que no conectan con los destinatarios ni tienen en cuenta sus prácticas; una difusión "neutral" que no toma partido y que creemos puede estar relacionada con la formación bibliotecaria apolítica que hemos recibido (Parada, 2017).

Para aportar desde el campo bibliotecario al mejoramiento del ecosistema de la comunicación científica, resulta interesante repensar nuestras prácticas y profundizar nuestros análisis, tomando prestadas teorías de otras áreas del saber. En ese sentido, los aportes conceptuales de los estudios sociales sobre la tecnología, en particular de la llamada "perspectiva socio-técnica" (Pinch y Bijker, 2008 [1987]) que propone una mirada más compleja sobre la construcción de artefactos, que ha sido aplicada por Guédon (2009) para el análisis de los repositorios. Sumado a eso, la experiencia acumulada en más de 15 años de construcción y seguimiento de repositorios institucionales por parte de las bibliotecas universitarias, nos llevan hoy a preguntarnos y valorar de otro modo la participación de todos los grupos relevantes de la comunicación de la ciencia en las instituciones. Del mismo modo, durante las etapas de planificación y construcción de estos artefactos no hemos habilitado la suficiente intervención de los investigadores y docentes, y tampoco hemos considerado en profundidad sus prácticas, circuitos y hábitos de difusión-publicación. Probablemente, todo eso constituya un factor más que influye en el escaso compromiso con el autoarchivo y con las políticas de acceso abierto?.

\section{La defensa del conocimiento como bien común frente a los servicios comerciales de información científica}

En el camino hacia el cumplimiento de la misión que las bibliotecas universitarias tienen de facilitar el acceso y la difusión de recursos de información y colaborar con la creación de conocimientos, tradicionalmente los bibliotecarios nos vinculamos con distintos proveedores de información científica para adquirir productos y servicios bibliográficos que atiendan las necesidades informativas de nuestra comunidad. A partir de la digitalización y del surgimiento del movimiento de acceso abierto, el rol de los proveedores y editores comerciales de la literatura científica empezó a sumar cuestionamientos, no sólo por los precios abusivos de sus productos digitales, sino además por imponer condiciones de suscripción retrógradas e injustas (compras por paquete, restricciones de uso y compartición de documentos, límites a la cantidad de copias simultáneas que se pueden "prestar" de un documento digital, no entrega de copias de resguardo si se cancela la suscripción, entre otras). En los últimos años esos cuestionamientos se multiplicaron y difundieron a través de declaraciones, manifiestos y campañas de firmas, llegando incluso a la suspensión de largos contratos entre bibliotecas y proveedores para forzar una negociación más beneficiosa para las instituciones (Fushimi, 2018a).

Por otra parte, la cobertura de estos productos y servicios bibliográficos de los que somos usuarios, y por los que en algunos casos pagamos onerosas cantidades de nuestros presupuestos, se relaciona con fenómenos más complejos que tienen que ver con la circulación del conocimiento, las relaciones de poder de la ciencia y los circuitos de circulación para la consagración académica (Beigel, 2018), los cuales, de alguna manera, el campo bibliotecológico ha contribuido a delinear. Actualmente,
9. Un estudio reciente realizado por Piwowar et al. (2018) estimó que solo el $27,9 \%$ del total de artículos con DOI se encontraban disponibles en la web en acceso abierto: el $4,8 \%$ en repositorios institucionales, el $3,2 \%$ en revistas de acceso abierto, el 3,6\% en revistas híbridas y un $16,2 \%$ en las páginas de los editores para consulta gratuita pero sin una licencia claramente abierta. Estos datos demuestran un lento avance del acceso abierto en general y también un pobre rol de los repositorios institucionales como infraestructura para alcanzar la meta del $100 \%$ de acceso abierto a la literatura tal como se había planteado inicialmente. 
10. Bradford trabajó en el Museo de Ciencias de Londres, estando a cargo de la sección química de la Biblioteca del Museo desde 1913, y dirigiéndola posteriormente hasta 1937.

11. La evaluación de la investigación basada únicamente en medidas como el factor de impacto han sido ampliamente criticadas en la Declaración de San Francisco sobre evaluación de la investigación (DORA 2012) y el Manifiesto de Leiden, hacia un mejor uso de los indicadores en la evaluación de la ciencia (2015). las dos bases de datos que determinan lo que se conoce como "corriente principal de la ciencia", o mainstream, son Web of Science de Clarivate Analytics -antes Thomsons Reuters y en sus inicios ISI, Institute for Scientific Information- con su tan conocido como criticado factor de impacto y Scopus de Elsevier con su ranking de revistas (Scimago Journal Rank). Ambas apoyan sus índices, y buena parte de sus criterios de inclusión, en la sumatoria de citas que reciben los trabajos publicados en sus revistas (cuantas más citas tenga, mejor será la revista, sin importar de cuál trabajo las obtiene) en un proceso que adjudica calidad y excelencia en base a ello. Los rankings de revistas se basan en la citación y en la ley de dispersión de la literatura científica definida por el bibliotecario Samuel C. Bradford en 1934, quien descubrió que cada disciplina tiene un núcleo básico y pequeño de revistas en las que se publica la mayor parte de la literatura especializada, mientras que el resto se publica en una enorme cantidad de revistas muy variadas e incluso de disciplinas diferentes. Pero cuando Bradford (1934) realizó su estudio, su objetivo era decidir qué revistas impresas suscribir en su biblioteca ${ }^{10}$ en un contexto de imposibilidad para obtenerlas a todas, y no determinar cuáles eran las mejores. Sin embargo, su análisis fue utilizado posteriormente para legitimar el prestigio de las publicaciones, y adoptado por los organismos de ciencia y técnica de todas las latitudes para evaluar la calidad de las producciones y también de los autores y sus carreras ${ }^{11}$.

Ante este panorama, como bibliotecarias y bibliotecarios comprometidos con el acceso a la información, gestores de repositorios institucionales y conocedores tanto de los indicadores bibliométricos más usados como de los problemas de cobertura y sesgos que poseen esas bases de datos, y de las prácticas de evaluación vigentes en el sistema científico, nos resulta al menos conflictivo seguir avalando el accionar de estos proveedores. También es problemático difundir y promover dentro de nuestras comunidades sus productos sin contextualizar su accionar. Con esto nos referimos a informar y concientizar acerca de los criterios según los cuales se incluyen -y excluyen- los contenidos allí disponibles, sus pretensiones de constituirse en las fuentes calificadas del saber científico universal, y ponderar su real aporte a cada disciplina. Observamos que nuestras capacitaciones sobre esas fuentes de información comerciales suelen transmitir, sin análisis ni crítica, los parámetros de evaluación de la calidad de la investigación con criterios exógenos, hecho que contribuye a legitimar y reforzar la lógica perversa impuesta por el sistema y el statu quo del núcleo central.

Del mismo modo, somos espectadores pasivos de la apropiación impaga (Zukerfeld y Kreimer, 2014) que se realiza con el conocimiento difundido en revistas y publicaciones locales o regionales alojados en repositorios, bibliotecas o incluso sitios web institucionales que disponen contenido de forma gratuita y que algunos proveedores comerciales incorporan en sus productos como forma de darle valor agregado a los suyos ${ }^{12}$. Paradójicamente, a su vez, condenamos en nuestro discurso otros sistemas de obtención de documentos como el difundido sitio Sci-Hub que apuesta a la apropiación del conocimiento por parte de la sociedad, transgrediendo las barreras de la propiedad intelectual para dar acceso gratuito y sin fines de lucro a los documentos necesarios para la enseñanza y la investigación.

Frente a las necesidades de nuestros usuarios para acceder a información científica en un sistema capitalista, como defensores del acceso al conocimiento inmersos en una realidad institucional y la función que la biblioteca universitaria asume en su comunidad, nos encontramos ante el dilema de si difundimos de forma acrítica estos sistemas, en los cuales invertimos parte del acotado presupuesto, o nos paramos del lado de la democratización y el acceso al conocimiento científico y enfocamos allí nuestros recursos y energía. Lo cual nos conduce, además, a reflexionar acerca de los repositorios como vía legítima prioritaria que nos permitirá alcanzar "el acceso abierto" y la necesidad de complementarlos con otras vías e infraestructuras para 
que la literatura científica esté cada vez más libre para todos. En este sentido, coincidimos con Guillermo Banzato (2019:9) cuando señala que debemos reconocer que el conocimiento como bien común requiere "un sistema de resguardo propio y anclado territorialmente en el Sur Global", así como también cuando plantea la necesidad de promover la "soberanía del conocimiento" para hacerle frente a las asimetrías y las inequidades del sistema hegemónico de comunicación de la ciencia. En sus palabras:

Se trata de defender nuestro derecho a crear sistemas que atiendan a la diversidad e idiosincrasia de nuestras sociedades, así como de la potestad de ofrecer para nuestros pueblos herramientas que les permitan desarrollar y difundir sus propios saberes (Banzato, 2019: 10).

En la convivencia necesaria con lo comercial es donde, para nosotras, existe una clara tensión ideológica, y en donde tenemos que ver cómo nos sumamos a construir un acceso abierto real que respete la tradición de América Latina en el desarrollo de circuitos regionales para la circulación abierta, colaborativa y no comercial del conocimiento (Babini, 2019). Ante las disyuntivas que hemos planteado, consideramos que debemos fortalecer nuestro rol de gestores de la información científica, tomando un papel más analítico y crítico de los recursos que usamos y difundimos. Por ejemplo, explicando en nuestros cursos y promoviendo el debate del significado de los principales índices bibliométricos y los riesgos que implica su uso descontextualizado. O bien, mostrando los orígenes y sesgos de cada una de las bases de datos que enseñamos a utilizar, así como el costo que implica poder acceder a ellas en relación con otros recursos de acceso abierto.

\section{Reflexiones finales}

La función social de nuestra profesión bibliotecaria nos condujo a sumarnos a la militancia por el libre acceso a la información científica. Lideramos de forma muy activa el proceso de construcción de los repositorios digitales en el ámbito de las bibliotecas universitarias y científicas en la Argentina, introduciendo de forma temprana el tema en la agenda del Estado nacional y promoviendo la sanción de la ley 26.899. De la misma manera, es destacable también el reclamo constante de nuestro colectivo en favor de excepciones para las bibliotecas en la ley de propiedad intelectual que nos permitan cumplir cabal y legalmente con nuestra función de ser proveedores del acceso a la información para fines educativos, culturales y científicos. Además, en las últimas décadas, hemos vivenciado el notable desarrollo y crecimiento académico de la Bibliotecología y Ciencia de la Información como disciplina y profesión en un contexto que modifica constantemente su objeto de estudio. Este hecho se refleja en la creación de posgrados específicos, la consolidación de la edición de revistas científicas especializadas junto con la aparición de otras nuevas, el crecimiento en la cantidad de encuentros, jornadas y congresos tanto profesionales como académicos en todo el país, así como la obtención creciente de becas y subsidios para la investigación por parte de nuestros graduados en el área (Liberatore, 2018).

Al tiempo que se va dando la consolidación del campo bibliotecológico nacional, enfrentamos dilemas como los planteados en este trabajo, y con frecuencia intentamos aplicar soluciones que creemos adecuadas porque provienen de contextos tradicionalmente mejor valorados, sin realizar un análisis previo que contemple las particularidades que se presentan en nuestro territorio, y tampoco pensamos que podríamos elaborar una respuesta propia. Creemos que muchas de estas actitudes tienen su origen en la formación que recibimos y que caracteriza nuestra disciplina: el perfil bajo y apolítico que hemos cultivado, focalizado en lo técnico y normalizado por códigos internacionales con origen en países anglosajones. 
Como comunidad bibliotecológica situada en la Argentina, debemos ganar autonomía, repensar cuál es nuestro rol en la sociedad, y evaluar de qué manera nuestras prácticas $\mathrm{y}$ actitudes contribuyen indirectamente al avance de las grandes corporaciones que comercializan un bien común como son el conocimiento y la cultura. Tenemos que cambiar nuestra postura acrítica y naturalizada sobre los productos comerciales y los sistemas externos de evaluación como verdades consagradas, porque a todas luces no favorecen la democratización del conocimiento científico. Debemos ser conscientes de que las decisiones que tomamos repercuten en nuestra comunidad y nos configuran como campo disciplinar, así como también, esforzarnos por contextualizar nuestras prácticas y escuchar a la comunidad a la que servimos.

Es necesario dejar atrás nuestro rol pseudoneutral y tomar partido para garantizar el acceso a la información científica. Ante las tensiones entre derechos contrapuestos, nuestra posición siempre debería estar del lado del acceso a la información. Solo así contribuiremos a la construcción de una sociedad más justa e inclusiva. Debemos tener un compromiso mayor: leer más, estudiar más, analizar más y no solo repetir el discurso que nos llega desde afuera, criticar lo que nos parece mal, opinar con fundamento, confrontar, discutir y promover el debate. Es tiempo de ser conscientes de la enorme responsabilidad que conlleva nuestro rol en el proceso de la comunicación científica y ejercerlo desde el lugar de agentes sociales de la democratización del conocimiento.

\section{Financiamiento}

Proyecto de investigación Repositorios digitales de acceso abierto en las universidades nacionales argentinas: desarrollo, evolución y perspectivas de futuro (Cod. Ho4o, período 2018-2019) que se desarrolló en el ámbito del Instituto de Investigaciones en Humanidades y Ciencias Sociales que depende de la Universidad Nacional de La Plata y el Consejo Nacional de Investigaciones Científicas y Técnicas (CONICET) de Argentina. 


\section{Q Referencias bibliográficas}

》 Argentina. Ley $\mathrm{N}^{\circ}$ 26.899. 2013. Repositorios digitales institucionales de acceso abierto. En Boletín Oficial de la República Argentina, 32.781, Primera Sección, 3-4. <http://servicios.infoleg.gob.ar/infoleglnternet/anexos/220000-224999/223459/norma.htm> [Consulta: 15 mayo 2020].

»Babini, Dominique. 2019. La comunicación científica en América Latina es abierta, colaborativa y no comercial. Desafíos para las revistas. En Palabra Clave. Vol. 8, no. 2, e065. <https://doi.org/10.24215/18539912e065>.

"Banzato, Guillermo. 2019. Soberanía del conocimiento para superar inequidades: políticas de Acceso Abierto para revistas científicas en América Latina. Mecila Working Paper Series, No. 18. <http://mecila.net/wp-content/uploads/2019/o8/WP18-Banzato-Online-Final.pdf> [Consulta: 15 mayo 2020].

" Beigel, Fernanda. 2018. Las relaciones de poder en la ciencia mundial. Un antiranking para conocer la ciencia producida en la periferia. En Nueva Sociedad. No. 274, 13-28. <https://nuso.org/media/articles/downloads/TG.Beigel_274. pdf>. [Consulta: 15 mayo 2020].

"Bohannon, John. 2016. Who's downloading pirated papers? Everyone. En Science. Vol. 352, no. 6285, 508-512. <https://doi.org/10.1126/science.352.6285.508>.

»Borrego, Ángel. 2017. Institutional repositories versus ResearchGate: The depositing habits of Spanish researchers. En Learned Publishing. Vol. 30, no. 3, 185192. <http://dx.doi.org/10.1002/leap.1099>.

»Bradford, Samuel C. 1934. Sources of information on specific subjects. En Engineering. A Illustrated Weekly Journal. Vol. 137, no. 3550, 85-86. Reprinted in 1985. Journal of Information Science. Vol. 10, no. 4, 176-18o. <do i:10.1177/016555158501000407>.

»Correa, Carlos M. 2009. Acceso a la cultura y derecho de propiedad intelectual: la búsqueda de un nuevo equilibrio. En ¿Desea guardar los cambios? Propiedad intelectual y tecnologías digitales: hacia un nuevo pacto social. Córdoba: Centro Cultural España. p. 130-152. <http://hipermedula.org/wp-content/ uploads/2010/10/desea_guardar_los_cambios.pdf> [Consulta: 15 mayo 2020].

"Declaración de México a favor del Ecosistema latinoamericano de Acceso Abierto No Comercial. 2017. <http://www.accesoabiertoalyc.org/declaracion-mexico/> [Consulta: 18 mayo 2020].

»Fushimi, Marcela. 2018a. Bloqueo y lobby en contra de las editoriales monopólicas. En Blog Acceso Abierto en movimiento. <http://accesoabierto.fahce.unlp. edu.ar/entradas/bloqueo-y-lobby-en-contra-de-las-editoriales-monopolicas> [Consulta: 15 mayo 2020].

"Fushimi, Marcela. 2018b. Desarrollo de repositorios digitales institucionales en las universidades nacionales en Argentina, período 2004-2015. Bernal: Universidad Nacional de Quilmes. 181 p. Tesis de maestría. <http://www.memoria.fahce. unlp.edu.ar/tesis/te.1629/te.1629.pdf> [Consulta: 15 mayo 2020].

"Guédon, Jean-Claude. 2009. It's a repository, it's a depository, it's an archive...: open access, digital collections and value. En ARBOR Ciencia, Pensamiento y Cultura. Vol. 185, no. 737, 581-595. <http://dx.doi.org/10.3989/arbor.2009.i737.315>. 
"IFLA. 2012. Código de Ética de IFLA para los bibliotecarios y trabajadores de la información. <https://www.ifla.org/files/assets/faife/codesofethics/spanishcodeofethicsfull.pdf> [Consulta: 15 mayo 2020].

»Laakso, Mikael y Juho Lindman. 2016. Journal copyright restrictions and actual open access availability - a study of articles published in eight top information systems journals (2010-2014). En Scientometrics. Vol. 109, no. 2, 1167-1189. $<$ https://doi.org/10.1007/s11192-016-2078-z>.

"Larivière, Vincent; Stefanie Haustein y Philippe Mongeon. 2015. The Oligopoly of Academic Publisher in the Digital Era. En PLOS One. Vol. 10, no. 6, eo127502. <https://doi.org/10.1371/journal.pone.0127502>.

»Liberatore, Gustavo. 2018. Visión actual del profesional de la Bibliotecología y Ciencia de la Información en la docencia y la investigación. En Jornadas de Docentes Investigadores Universitarios en Ciencia de la Información (2a: 2018: Mar del Plata). p. 7-17.

" Machin-Mastromatteo, Juan de.; Alejandro Uribe-Tirado y María E. RomeroOrtiz. 2016. Piracy of scientific papers in Latin America. An analysis of Sci-Hub usage data. En Information development. Vol. 32, no. 5, 1806-1814. <https://doi. org/10.1177/0266666916671080>.

» Miguel, Sandra; Claudia M. González y Edgardo Ortiz-Jaureguizar. 2018. Preferencias de investigadores y prácticas institucionales/disciplinares en la difusión y socialización de los resultados de investigación. En Información, cultura y sociedad. No. 38, 53-76. <https://doi.org/10.34096/ics.i38.3989>.

"Open Letter on Intellectual Property and COVID-19 to the Director General of WIPO. 2020. <https://www.ifla.org/ES/node/92993>. [Consulta: 15 mayo 2020].

»Parada, Alejandro. 2017. Endogamia y Bibliotecología/Ciencia de la Información. En Información, Cultura y Sociedad. No. 36, 5-10. <http://revistascientificas.filo.uba.ar/index.php/ICS/article/view/3551> [Consulta: 15 mayo 2020].

»Pinch, Trevor J. y Wiebe. E. Bijker. 2008 [1987]. La construcción social de hechos y artefactos: o acerca de cómo la sociología de la ciencia y la sociología de la tecnología pueden beneficiarse mutuamente. En Thomas, Hernán y Alfonso Buch, coords. Actos, actores y artefactos: Sociología de la tecnología. Bernal: Universidad Nacional de Quilmes. p. 19-62.

» Piwowar, Heather; Jason Priem; Vincent Larivière; Juan Pablo Alperin; Lisa Matthias; Bree Norlander; Ashley Farley; Jevin West y Stefanie Haustein. 2018. The state of OA: a large-scale analysis of the prevalence and impact of Open Access articles. En PeerJ, 6, e4375. <https://doi.org/10.7717/peerj.4375>.

"Sanllorenti, Ana María; Lucía Pelaya y Martín Williman. 2012. Instrumentos para la gestión del derecho de autor en repositorios de Acceso Abierto. En Revista Interamericana de Bibliotecología. Vol. 34, no. 3, 313-328. < https://revistas.udea. edu.co/index.php/RIB/article/view/11969 > [Consulta: 15 mayo 2020].

"Swartz, Aaron. 2012 [2008]. Manifiesto por la Guerrilla del Acceso Abierto. $<$ https://endefensadelsl.org/guerrilla_del_acceso_abierto.html> [Consulta: 15 mayo 2020].

»Unzurrunzaga, Carolina. 2017. Hábitos de los investigadores de Humanidades y Ciencias Sociales para visibilizar sus publicaciones en el entorno digital. Un estudio de caso argentino. Salamanca: Universidad de Salamanca. 100 p. Trabajo de Fin de Máster en Sistemas de Información Digital, curso 2016-2017. <http://hdl. handle.net/10366/138126> [Consulta: 15 mayo 2020]. 
»Zukerfeld, Mariano y Pablo Kreimer. 2014. La explotación cognitiva: tensiones emergentes en la producción y uso social de conocimientos científicos tradicionales, informacionales y laborales. En Kreimer, Pablo; Hebe Vessuri; Léa Velho y Antonio Arellano, coords. Perspectivas latinoamericanas en el estudio social de la ciencia, la tecnología y el conocimiento. Buenos Aires: Siglo XXI. p. 178-193. 
